\title{
Prevalência do desvio fonológico em crianças da cidade de Salvador, Bahia
}

\author{
Prevalence of phonological disorders in children from \\ Salvador, Bahia, Brazil
}

\author{
Laura Giotto Cavalheiro ${ }^{1}$, Ana Rita Brancalioni², Márcia Keske-Soares ${ }^{3}$
}

\begin{abstract}
RESUMO
Objetivo: Verificar a prevalência de desvio fonológico conforme idade, gênero e nível sócio econômico de crianças da cidade de Salvador, Bahia, Brasil. Métodos: A amostra foi composta por 2880 crianças de ambos os gêneros, na faixa etária de 4 anos a 6 anos e 11 meses, de nível socioeconômico baixo, médio e alto, matriculadas nas escolas municipais de Salvador. Inicialmente, todas as crianças foram submetidas, de forma individual, à triagem fonoaudiológica e triagem auditiva. Além disso, foi realizada uma anamnese com os responsáveis e uma entrevista com os professores. Em seguida, foi realizada a avaliação fonológica e análise contrastiva, a fim de diagnosticar crianças com desvio fonológico. Calculou-se a prevalência do desvio fonológico e foi realizado tratamento estatístico. Resultados: A prevalência do desvio fonológico foi de 9,17\%. Verificou-se maior prevalência do desvio fonológico no gênero masculino. Além disso, ao associar os gêneros às variáveis "idade" e "nível socioeconômico", a prevalência variou estatisticamente. Conclusão: Fatores biológicos e sociais podem influenciar na aquisição das habilidades fonológicas da linguagem oral. Medidas de prevenção e ações voltadas para identificar e tratar o desvio fonológico devem considerar as diferentes classes de desenvolvimento socioeconômico.
\end{abstract}

Descritores: Distúrbios da fala; Prevalência; Criança; Fala; Desenvolvimento infantil; Classe social

\section{INTRODUÇÃO}

A prevalência é o número de pessoas, em uma determinada população, que tem uma característica, em saúde, normalmente uma doença, em um determinado ponto do tempo (geralmente o tempo em que um inquérito é feito) $)^{(1)}$. Estudos epidemiológicos não oferecem a causa da doença, mas os índices encontrados auxiliam na avaliação das necessidades e do planejamento dos serviços de saúde, permitindo compreender o quanto é comum, ou rara, uma determinada doença ou situação numa população( ${ }^{(2)}$.

Trabalho realizado Programa de Pós-Graduação em Distúrbios da Comunicação Humana, Universidade Federal de Santa Maria - UFSM - UFSM (RS), Brasil.

Conflito de interesses: Não

(1) Programa de Pós-graduação (Mestrado) em Distúrbios da Comunicação Humana, Universidade Federal de Santa Maria - UFSM - Santa Maria (RS), Brasil.

(2) Programa de Pós-graduação (Doutorado) em Distúrbios da Comunicação Humana, Universidade Federal de Santa Maria - UFSM - Santa Maria (RS), Brasil.

(3) Curso de Fonoaudiologia e Programa de Pós-Graduação em Distúrbios da Comunicação Humana, Universidade Federal de Santa Maria - UFSM Santa Maria (RS), Brasil.

Endereço para correspondência: Laura Giotto Cavalheiro. Al. Praia de Guaratuba, 1575/11, Stella Mares, Salvador (BA), Brasil, CEP: 41600-270. E-mail: laufono@uol.com.br

Recebido em: 5/10/2011; Aceito em: 5/6/2012
As estimativas de prevalência de distúrbios de fala na infância modificam-se de acordo com o método utilizado e quanto maior o número amostral maior fidedignidade haverá no índice epidemiológico encontrado ${ }^{(3)}$. Para algumas crianças, o processamento das informações fonológicas pode acontecer de maneira diferente do esperado. Quando essa diferença é caracterizada por uma desorganização ou inadaptação no sistema de sons da criança em relação ao sistema padrão de sua comunidade linguística, sem a presença de qualquer comprometimento orgânico, tem-se o denominado desvio fonológico ${ }^{(4)}$.

De acordo com a literatura ${ }^{(3-5)}$, o quadro clínico do desvio fonológico é representado por fala espontânea ininteligível em idade superior a quatro anos, audição normal, capacidades de linguagem expressiva e compreensiva bem desenvolvidas e ausência de qualquer comprometimento orgânico. $\mathrm{O}$ desvio fonológico pode envolver tanto um número limitado de sons da fala quanto distúrbios mais graves, envolvendo vários sons, e que resultam em inteligibilidade reduzida ${ }^{(6)}$.

Estudo específico ${ }^{(7)}$ salienta que obter a prevalência de alterações fonológicas contribui para a criação de projetos de prevenção e de intervenção fonoaudiológica na área de linguagem. Além disso, conhecer as variáveis que podem influenciar na prevalência de determinada patologia favorece $o$ desenvolvimento de ações preventivas. Diante disso, o objetivo 
deste estudo foi verificar a prevalência de desvio fonológico e associá-lo às variáveis referentes à idade, ao gênero e ao nível sócio econômico, em crianças da cidade de Salvador, Bahia (BA).

\section{MÉTODOS}

Esta pesquisa é de caráter transversal e do tipo quantitativa. Foi realizada a partir de coleta de dados de projeto de pesquisa devidamente aprovado pelo Comitê de Ética em Pesquisa da Universidade Federal de Santa Maria, sob número 23081.006440/2009-60. Os responsáveis assinaram o Termo de Consentimento Livre e Esclarecimento (TCLE), conforme Resolução CNS 196/96. Além disso, houve assinatura do Termo de Autorização Institucional por parte das escolas participantes.

Inicialmente, para classificação do nível socioeconômico foi realizado estudo estatístico baseado no Índice de Desenvolvimento Socioeconômico - IDESE ${ }^{(8)}$, considerando os indicadores de desenvolvimento apresentados no Anuário da cidade de Salvador de 2003, a fim de estabelecer em quais regiões seriam realizadas a coleta de dados. Depois de calculado o IDESE ${ }^{(8)}$ foi realizado sorteio aleatório de uma região para cada nível socioeconômico (baixo, médio e alto), bem como das escolas municipais que atendiam a população de crianças de 4 a 6 anos de idade que compreendiam as regiões selecionadas.

Para delimitar a amostra, estimando-se uma confiança de $95 \%$ e margem de erro de $5 \%$, por meio do cálculo de amostragem estratificada proporcional ao tamanho dos grupos anteriormente referidos da população, deveriam fazer parte da pesquisa 950 crianças na faixa etária de 4 anos e 0 mês a 4 anos e 11 meses; 952 de 5 anos e 0 mês a 5 anos e 11 meses; e 955 de 6 anos e 0 mês a 6 anos e 11 meses. Assim, foram constituídos três grupos de estudo.

Entretanto, a fim de favorecer a comparação entre os grupos foi estabelecido por convenção que cada faixa etária teria o mesmo número de sujeitos. Desta forma, para cada faixa etária foram selecionadas 960 crianças. Para contemplar os objetivos específicos deste estudo, que envolviam a verificação da prevalência não só considerando a faixa etária, mas também gênero e nível socioeconômico, foi realizada uma nova convenção para constituição da amostra a ser selecionada. Assim, para cada grupo de 960 crianças, considerando-se a faixa etária, havia 480 do gênero masculino e 480 do feminino, e 320 do nível socioeconômico baixo, 320 do nível médio e 320 do nível alto. O número total foi de 2880 crianças.

Com a finalidade de evitar qualquer viés durante a análise dos resultados foi estabelecido que a coleta acontecesse com 1200 crianças de cada faixa etária. Após a coleta de dados, para finalmente constituir o grupo selecionado, foi realizado sorteio aleatório de 960 crianças para cada faixa etária, seguindo também os determinantes gênero e nível socioeconômico.

Para eleição destas 1200 crianças foi solicitado às escolas que se encontravam nas regiões distritais de diferentes níveis de desenvolvimento socioeconômico a entrega da lista de alunos matriculados que se enquadravam na faixa etária proposta pelo estudo. A partir desta lista, foram convocados, conforme ordem alfabética, o responsável de cada criança para esclarecimentos sobre o estudo e obtenção de consentimento para participar da pesquisa. No total, foram avaliadas 3600 crianças que cursavam as séries pré-escolares da rede pública municipal de ensino do município de Salvador. Entretanto, foram incluídas na amostra 2880 crianças, na faixa etária de 4 anos e 0 mês a 6 anos e 11 meses, de ambos os gêneros.

Foram adotados como critérios de exclusão: a) alunos cujos pais e/ou responsáveis não autorizaram a participação no estudo; b) alunos que não completaram todas as avaliações, por não comparecerem por três vezes consecutivas; c) alunos que estavam utilizando medicações psicoativas, por entender que estas poderiam interferir nos resultados das avaliações; d) alunos que apresentaram alterações na triagem auditiva; e) alunos em tratamento psicológico, neurológico ou psiquiátrico; f) alunos que apresentavam alterações neurológicas e psicológicas significativas ou síndromes, informadas durante a anamnese com pais e entrevista com os professores.

Este estudo foi realizado em dois momentos distintos. No primeiro momento, todas as crianças foram submetidas, de forma individual, à triagem fonoaudiológica e à triagem auditiva. Além disso, foi realizada uma anamnese, com os responsáveis e uma entrevista com os professores. Em um segundo momento, foi realizada a Avaliação Fonológica da Criança ${ }^{(9)}$, sendo realizada a análise contrastiva.

A partir dos resultados da triagem fonoaudiológica e da avaliação fonológica foram identificadas as crianças que apresentavam desvio fonológico segundo critério da literatura para diagnóstico de desvio fonológico ${ }^{(3-5)}$. Em seguida, os dados foram tabulados em planilha eletrônica e submetidos a tratamento estatístico por meio do teste Qui-quadrado, considerando significância de 5\% $(\mathrm{p}<0,05)$.

\section{RESULTADOS}

Verificou-se que a prevalência de desvio fonológico foi de $9,17 \%$ ( $\mathrm{n}=264$ crianças) da população total ( 2880 crianças). A idade de 5 anos apresentou maior prevalência $(9,48 \%)$ quando comparada às faixas etárias de 4 anos $(8,96 \%)$ e 6 anos $(9,06 \%)$ porém sem diferença entre elas. Logo, estes resultados sugerem que não há um padrão de aumento ou diminuição do desvio fonológico conforme a faixa etária (Figura 1).

A prevalência do desvio fonológico para o gênero masculino foi maior $(13,3 \%)$ que para o gênero feminino $(5,0 \%)$,

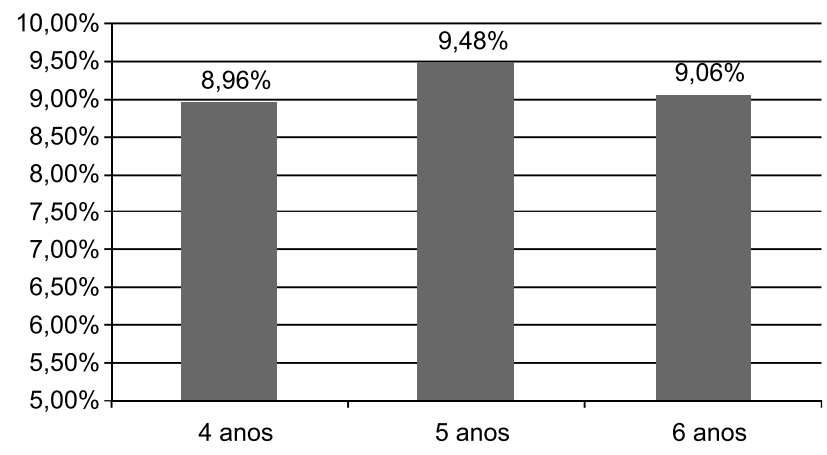

Teste Qui-quadrado $(p=0,9162)$

Figura 1. Prevalência de desvio fonológico conforme a idade 
havendo diferença entre eles. Assim, os dados encontrados permitiram verificar razão de 2,7 casos de desvio fonológico do gênero masculino para cada caso do gênero feminino (Figura 2).

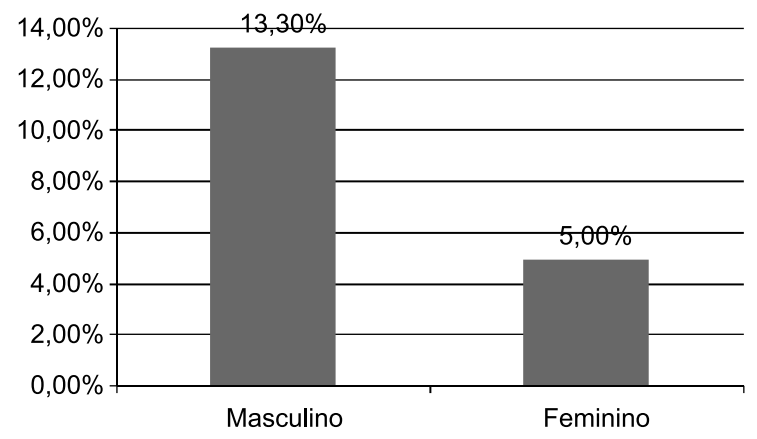

Teste Qui-quadrado $(p=0,0001)$

Figura 2. Prevalência de desvio fonológico conforme o gênero

O nível socioeconômico médio apresentou maior prevalência $(9,69 \%)$ quando comparado aos níveis baixo $(8,75 \%)$ e alto $(9,06 \%)$. Entretanto, não houve diferença entre eles (Figura 3).

As crianças com nível socioeconômico médio apresentaram maiores prevalências em cada grupo de idade. No entanto, nenhuma destas diferenças foi significativa (Tabela 1).

Houve menor prevalência de desvio fonológico para a idade de 5 anos $(11,25 \%)$ quando comparada com as idades de 4 e 6 anos $(14,38 \%, 14,38 \%)$, sendo esta diferença significativa. Além disso, observou-se que no gênero masculino e na idade de 5 anos, o nível socioeconômico alto apresentou prevalência de alteração fonológica inferior $(4,38 \%)$ aos níveis

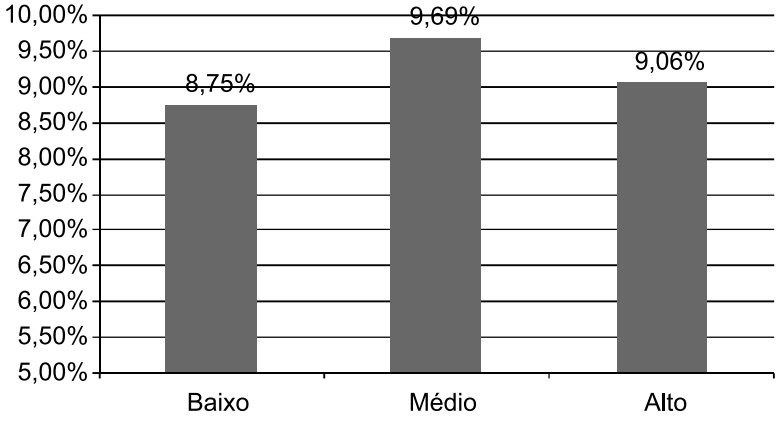

Teste Qui-quadrado $(\mathrm{p}=0,9042)$

Figura 3. Prevalência de fesvio fonológico conforme o nível socioeconômico

baixo $(14,38 \%)$ e médio $(15,00 \%)$ havendo diferença entre eles (Tabela 2).

Observou-se maior prevalência de desvio fonológico para a idade de 5 anos $(7,71 \%)$ quando comparada com as idades de $4(3,13 \%)$ e 6 anos $(3,75 \%)$, havendo diferença entre elas. Além disso, verificou-se que no gênero feminino e na idade de 5 anos, o nível socioeconômico alto apresentou prevalência de alteração fonológica superior $(13,75)$ aos níveis baixo $(4,38 \%)$ e médio $(3,75 \%)$, havendo diferença entre eles (Tabela 3 ).

\section{DISCUSSÃO}

A prevalência de desvio fonológico encontrada apresentou percentual próximo aos índices encontrados em outros estudos internacionais ${ }^{(5,10-13)}$. Entre esses estudos, encontraram-se os da população americana com prevalência em $10 \%^{(10)}$, da

Tabela 1. Prevalência de desvio fonológico de acordo com o nível socioeconômico e a idade

\begin{tabular}{|c|c|c|c|c|c|c|c|c|c|c|c|c|}
\hline \multirow{3}{*}{$\begin{array}{l}\text { Nível socio- } \\
\text { econômico }\end{array}$} & \multicolumn{9}{|c|}{ Idade } & \multirow{2}{*}{\multicolumn{3}{|c|}{ Total }} \\
\hline & \multicolumn{3}{|c|}{4 anos } & \multicolumn{3}{|c|}{5 anos } & \multicolumn{3}{|c|}{6 anos } & & & \\
\hline & $\begin{array}{l}\text { População } \\
\text { do estudo } \\
\text { (N) }\end{array}$ & $\begin{array}{l}\text { Casos de } \\
\text { DF (n) }\end{array}$ & $\begin{array}{c}\text { Prevalência } \\
\text { (\%) }\end{array}$ & $\begin{array}{l}\text { População } \\
\text { do estudo } \\
\text { (N) }\end{array}$ & $\begin{array}{l}\text { Casos de } \\
\text { DF (n) }\end{array}$ & $\begin{array}{c}\text { Prevalência } \\
\text { (\%) }\end{array}$ & $\begin{array}{l}\text { População } \\
\text { do estudo } \\
\text { (N) }\end{array}$ & $\begin{array}{c}\text { Casos de } \\
\text { DF (n) }\end{array}$ & $\begin{array}{c}\text { Prevalência } \\
\text { (\%) }\end{array}$ & $\begin{array}{l}\text { População } \\
\text { do estudo } \\
\text { (N) }\end{array}$ & $\begin{array}{c}\text { Casos de } \\
\text { DF (n) }\end{array}$ & $\begin{array}{c}\text { Prevalência } \\
(\%)\end{array}$ \\
\hline Baixo & 320 & 26 & 8,13 & 320 & 31 & 9,69 & 320 & 27 & 8,44 & 960 & 84 & $8,75^{\#}$ \\
\hline Médio & 320 & 31 & 9,69 & 320 & 31 & 9,69 & 320 & 31 & 9,69 & 960 & 93 & $9,69^{\#}$ \\
\hline Alto & 320 & 29 & 9,06 & 320 & 29 & 9,06 & 320 & 29 & 9,06 & 960 & 87 & $9,06^{\#}$ \\
\hline Total & 960 & 86 & $8,96^{\#}$ & 960 & 91 & $9,48^{\#}$ & 960 & 87 & $9,06^{\#}$ & 2880 & 264 & $9,17^{\#}$ \\
\hline
\end{tabular}

Teste Qui-quadrado $(p<0,05)$

\# Valores sem diferença significativa

Legenda: DF = desvio fonológico

Tabela 2. Prevalência de desvio fonológico de acordo com o nível socioeconômico e a idade no gênero masculino

\begin{tabular}{|c|c|c|c|c|c|c|c|c|c|c|c|c|}
\hline \multirow{3}{*}{$\begin{array}{l}\text { Nível socio- } \\
\text { econômico }\end{array}$} & \multicolumn{9}{|c|}{ Idade } & \multirow{2}{*}{\multicolumn{3}{|c|}{ Total }} \\
\hline & \multicolumn{3}{|c|}{4 anos } & \multicolumn{3}{|c|}{5 anos* } & \multicolumn{3}{|c|}{6 anos } & & & \\
\hline & $\mathrm{N}=480$ & $\mathrm{n}=69$ & $P=14,38$ & $\mathrm{~N}=480$ & $\mathrm{n}=54$ & $P=11,25$ & $\mathrm{~N}=480$ & $\mathrm{n}=69$ & $P=14,38$ & $\mathrm{~N}=1440$ & $n=192$ & $P=13,33$ \\
\hline Baixo & 160 & 21 & 13,13 & 160 & 23 & 14,38 & 160 & 24 & 15,00 & 480 & 68 & 14,17 \\
\hline Médio & 160 & 26 & 16,25 & 160 & 24 & 15,00 & 160 & 22 & 13,75 & 480 & 72 & 15,00 \\
\hline Alto* & 160 & 22 & 13,75 & 160 & 7 & 4,38 & 160 & 23 & 14,38 & 480 & 52 & 10,83 \\
\hline
\end{tabular}

* Valores significativos $(p<0,05)$ - Teste Qui-quadrado

Legenda: $\mathrm{N}$ = número da população do estudo; $\mathrm{n}$ = número de casos de desvio fonológico; $\mathrm{P}=$ prevalência de desvio fonológico (em \%) 
Tabela 3. Prevalência de desvio fonológico de acordo com o nível socioeconômico e a idade no gênero feminino

\begin{tabular}{|c|c|c|c|c|c|c|c|c|c|c|c|c|}
\hline \multirow{3}{*}{$\begin{array}{l}\text { Nível socio- } \\
\text { econômico }\end{array}$} & \multicolumn{9}{|c|}{ Idade } & \multirow{2}{*}{\multicolumn{3}{|c|}{ Total }} \\
\hline & \multicolumn{3}{|c|}{4 anos } & \multicolumn{3}{|c|}{5 anos* } & \multicolumn{3}{|c|}{6 anos } & & & \\
\hline & $\mathrm{N}=480$ & $\mathrm{n}=17$ & $P=3,54$ & $\mathrm{~N}=480$ & $\mathrm{n}=37$ & $P=7,71$ & $\mathrm{~N}=480$ & $\mathrm{n}=18$ & $P=3,75$ & $N=1440$ & $\mathrm{n}=72$ & $P=5,00$ \\
\hline Baixo & 160 & 5 & 3,13 & 160 & 8 & 5,00 & 160 & 3 & 1,88 & 480 & 16 & 3,33 \\
\hline Médio & 160 & 5 & 3,13 & 160 & 7 & 4,38 & 160 & 9 & 5,63 & 480 & 21 & 4,38 \\
\hline Alto* & 160 & 7 & 4,38 & 160 & 22 & 13,75 & 160 & 6 & 3,75 & 480 & 35 & 7,29 \\
\hline
\end{tabular}

* Valores significativos $(p<0,05)$ - Teste Qui-quadrado

Legenda: $\mathrm{N}$ = número da população do estudo; $\mathrm{n}$ = número de casos de desvio fonológico; $\mathrm{P}$ = prevalência de desvio fonológico (em \%)

população cubana com prevalência de $12 \%^{(11)}$, da população italiana com prevalência de $15 \%{ }^{(12,13)}$, e da população chinesa com prevalência de $16 \% \%^{(5)}$.

No entanto, considerando a literatura nacional, a prevalência encontrada apenas foi semelhante a um estudo ${ }^{(14)}$ que encontrou $10 \%$ de alterações fonológicas em crianças da faixa etária de 5 anos e quatro meses a 6 anos e 11 meses, na cidade de Porto Alegre-RS. Na maior parte dos estudos que verificaram a prevalência de desvios fonológicos na população brasileira, os índices de prevalências encontrados foram superiores. Estudos nacionais apontaram, por exemplo, índices de $18,55 \%{ }^{(15)}, 27^{(16)}$ e $34,16 \%{ }^{(17)}$.

É importante considerar que a diferença encontrada entre os percentuais das prevalências mencionadas pode ser justificada pelo fato de tais estudos ${ }^{(15-17)}$ incluírem um número de sujeitos inferior ao da presente pesquisa e, nestas situações, o viés de seleção e da "variável de influência". Tal variável poderia favorecer um maior número de autorização por parte dos pais das crianças com alterações de fala, pelo fato de estarem mais sensíveis à aceitação com o intuito de receber ajuda para o caso ${ }^{(15)}$.

Esta mesma justificativa pode explicar a proximidade dos índices de prevalência encontrados no presente estudo quando comparado os estudos internacionais ${ }^{(5,10-13,18)}$, nos quais as populações estudadas são maiores e mais abrangentes. Entretanto, esta grande variabilidade de percentuais encontradas na literatura também pode ocorrer devido à improbabilidade de afirmar com exatidão quantas pessoas têm distúrbios de comunicação específicos, visto que as pesquisas utilizaram diversas definições de distúrbios, em populações-alvo diferentes $^{(19)}$. Esta informação pode ser elucidada ao verificar os dados do estudo realizado em 2006 na região sul do país ${ }^{(15)}$ que permitiu a presença de até duas alterações miofuncionais em crianças com desvio fonológico, diferentemente do que foi aplicado neste estudo, que excluiu toda e qualquer alteração miofuncional que pudesse interferir nos resultados de fala.

Ao ser analisada a prevalência do desvio fonológico nas diferentes faixas etárias, o fato de a idade não influenciar a prevalência do desvio é um achado importante, pois permite afirmar que estas crianças, na faixa etária de 4 anos, já apresentam a aquisição fonológica completa, informação que corrobora dados descritos por outros autores ${ }^{(4,9)}$.

Esta interpretação é possível, porque, à medida que a criança adquire regras fonológicas mais importantes, mantendo os contrastes, aumenta a inteligibilidade de sua fala. Assim, como não houve decréscimo no prejuízo fonológico evidenciado quando comparadas as faixas etárias, é possível afirmar que as habilidades fonológicas estejam estabelecidas por volta de 4 anos. Portanto, os dados obtidos demonstram presença de desvios fonológicos em crianças que já deveriam estar com sistema fonológico adquirido ${ }^{(20)}$.

Quanto à prevalência em relação ao gênero, os achados apontam que o gênero masculino influencia significativamente a prevalência do desvio fonológico. Tal achado concorda com outros estudos ${ }^{(3,7,15,17,21)}$ que verificaram haver maior incidência de desvio fonológico no gênero masculino. Porém, os achados diferem de outras pesquisas ${ }^{(22,23)}$, que ao utilizarem escalas de desenvolvimento para identificação de atrasos no desenvolvimento da fala, verificaram maior prevalência de alterações de fala em sujeitos do gênero feminino.

O fato de a variável nível socioeconômico não influenciar estatisticamente na prevalência do desvio fonológico corrobora achados de outro estudo ${ }^{(18)}$. Entretanto, discorda de outros, os quais verificaram haver influência da situação socioeconômica no processo de desenvolvimento fonológico ${ }^{(24-27)}$, sendo o nível de desenvolvimento social e econômico baixo indicado como determinante para aumentar o risco de alterações na fala ${ }^{(25,26)}$.

Os dados do presente estudo, apesar de não corroborarem os achados propostos pela literatura, alertam que o desenvolvimento socioeconômico nem sempre é determinante para promover atrasos no desenvolvimento infantil. Desta forma, é importante estabelecer propostas de promoção de saúde que considerem não apenas as classes sociais menos privilegiadas, mas também as demais classes de desenvolvimento socioeconômico. Esta preocupação está de acordo com as informações oferecidas por estudo epidemiológico que afirma que o crescimento da epidemiologia social possibilita o entendimento da influência ou não do meio físico e social no estado de saúde, facilitando a coordenação de ações para objetivos comuns e abrindo novos caminhos no campo da saúde pública em direção a uma população mais saudável ${ }^{(28)}$.

Quando considerado o gênero separadamente, conforme a idade e o nível socioeconômico, a prevalência encontrada para tais variáveis foi diferente. Dessa forma, pode-se inferir que a divergência quanto à prevalência do desvio fonológico de acordo com o gênero, nas pesquisas ${ }^{(7,15,17,21,22,23)}$ que não consideraram a análise do nível socioeconômico, pode ter ocorrido devido à seleção da amostra. Assim, alguns estudos podem ter enfocado populações de níveis socioeconômicos mais baixos e, portanto, ter sido encontrada maior prevalência no gênero masculino ${ }^{(7,5,17,21)}$, ou então enfocado populações de nível sócio econômico mais alto e, com isso, identificado 
maior prevalência de desvio fonológico em sujeitos do gênero feminino ${ }^{(22,23)}$.

\section{CONCLUSÃO}

A prevalência de desvio fonológico encontrada na cidade de Salvador-BA, de 9,17\%, é inferior à prevalência apontada por estudos realizados em outras regiões do país. Considerando as variáveis "idade", "gênero" e "nível socioeconômico", analisadas isoladamente, pode-se concluir que apenas a variável gênero interfere significativamente na prevalência do desvio fonológico, sendo este mais prevalente no gênero masculino.

Entretanto, ao associar as variáveis "idade" e "nível socioeconômico", de acordo com o gênero, a prevalência variou estatisticamente. Diante disso, pode-se inferir que fatores biológicos e sociais podem influenciar na aquisição das habilidades fonológicas da linguagem oral. Por isso, as medidas de prevenção e ações voltadas para identificar e tratar o desvio fonológico devem considerar não apenas as classes sociais menos privilegiadas, mas também as demais classes de desenvolvimento socioeconômico.

\begin{abstract}
Purpose: To verify the prevalence of phonological disorders according to age, gender and socioeconomic level of children from Salvador, Bahia, Brazil. Methods: The sample comprised 2880 children of both genders from low, middle and high socioeconomic levels enrolled in public schools from Salvador, with ages ranging from 4 years to 6 years and 11 months. Initially, all children were individually screened for speech-language and hearing disorders. Moreover, an anamnesis was conducted with caregivers/parents, and teachers answered to an interview. After that, a phonological assessment and a contrastive analysis were conducted, in order to diagnose children with phonological disorders. The prevalence of phonological disorder was calculated, and statistical analyses were performed. Results: The prevalence of speech disorders was 9.17\%. It was verified a higher prevalence of phonological disorders for male subjects. In addition, when genders were associated to the variables "age" and "socioeconomic status", the prevalence varied statistically. Conclusion: Biological and social factors might influence the acquisition of phonological skills of oral language. Prevention measures and actions directed to identifying and treating phonological disorders should consider the different classes of socioeconomic development.
\end{abstract}

Keywords: Speech disorders; Prevalence; Child; Speech; Child development; Social class

\title{
REFERÊNCIAS
}

1. Jekel JF, Katz DL, Elmore JG. Epidemiologia, bioestatística e medicina preventiva. $2^{\mathrm{a}}$ ed. São Paulo: Artmed; 2005. p. 207

2. Beaglehole R, Bonita R, Kjellström T. Epidemiologia básica. São Paulo: Santos; 2003. 175p.

3. Shriberg LD, Tomblin JB, McSweenty JL. Prevalence of speech delay in 6-year-old children and comorbidity with language impairment II. J Speech Lang Hear Res. 1999;42(6):1482-98.

4. Vieira MG, Mota HB, Keske-Soares M. Relação entre idade, grau de severidade do desvio fonológico e consciência fonológica. Rev Soc Bras Fonoaudiol. 2004;9(3):144-50.

5. Siok WT, Perfetti CA, Jin Z, Tan LH. Biological abnormalities of impaired reading in constrained by culture. Nature. 2004;431(7004):716.

6. Sices L, Taylor G, Freebairn L, Hansen A, Lewis B. Relationship between speech-sound disorders and early literacy skills in preschoolage children: impact of comorbid language impairment. J Dev Behav Pediatr. 2007;28(6):438-47.

7. Patah LK, Takiuchi N. Prevalência das alterações fonológicas e uso dos processos fonológicos em escolares aos 7 anos. Rev CEFAC. 2008;10(2):158-67.

8. Fundação de Economia e Estatística (FEE) [Internet] [acesso em mar 2011]. Disponível em: http://www.fee.tche.br/sitefee/pt/content/ estatisticas/pg_idese.php.

9. Yavas MS, Hernandorena CL, Lamprecht RR. Avaliação Fonológica da Criança. 2a ed. Porto Alegre: Artes Médicas, 2002.

10. Gierut J. Treatment efficacy: functional phonological disorders in children. J Speech Lang Hear Dis. 1998;41(1):85-100.

11. Quintana TP, Tamayo IV, Blanco NL, Carvajal FU, Alvarez MR. Valoración logofoniátrica de niñas y niños antes de iniciar el círculo infantil. Rev Cubana Pediatr. 2003;75(3)

12. Longoni AM, Richardison JT, Aiello A. Articulatory rehearsal and phonological storage in working memory. Mem Cognit. 1993;21(1):11-22.

13. Longoni AM, Aiello A. Working memory. Mem and Cognit. 2004:37(1)34-45.

14. Nacente VP, França MP. Estudo da prevalência de alterações na aquisição fonológica em pré-escolares e escolares. Fono Brasil. 2005;3(1):1-4

15. Casarin MT. Prevalência dos desvios de fala em pré-escolares de escolas públicas estaduais de Santa Maria-RS [dissertação]. Santa Maria: Universidade Federal de Santa Maria; 2006.

16. Cigana L, Chiari B, Motta HB, Cechella C. Perfil do desenvolvimento fonológico de crianças de creches da rede municipal de Santa Maria RS, na faixa etária de 4:0 a 6:2 anos. Pró-Fono. 1995;7(2):15-20.

17. Silva EI, Lima EM, Silveira PC. Ocorrência de desvios fonológicos em crianças de escolas públicas do município de Camaragibe. Fono Atual. 2003;6(25):4-12.

18. Keating D, Turrel G, Ozanne A. Childhood speech disorders: reported prevalence, comorbidity and socioeconomic profile. J Pediatr Child Health. 2001;37(5):431-6.

19. Riper CV, Emerick L. Correção da linguagem: uma introdução à patologia da fala e à audiologia. Porto Alegre: Artes Médicas; 1997. 445p.

20. Pagliarin KC, Brancalioni AR, Keske-Soares M, Souza AP. Relação entre gravidade do desvio fonológico e fatores familiares. Rev CEFAC. 2011;13(3):414-27.

21. Farias SR, Ávila CR, Vieira MM. Relação entre fala, tônus e praxia não-verbal do sistema estomatognático em pré-escolares. Pró-Fono. 2006;18(3):267-76. 
22. Van Hulle CA, Goldsmith S, Lemery KS. Genetic, environmental, and gender effects on individual differences in toddler expressive language. J Speech Lang Hear Res. 2004;47(4):904-12.

23. Shevell MI, Majnemer A, Webster RI, Platt RW, Birnbaum R. Outcomes at school age of preschool children with developmental language impairment. Ped Neurol. 2005;32(4):264-9.

24. Mello DE. A interferência da situação socioeconômica no processo do desenvolvimento fonológico em crianças de classes sociais e idades diferentes. Língua \& Letras. 2002;1:75-96.

25. Rezende MA, Lima FG, Beteli VC, Santos JL. Habilidades de linguagem e pessoal-social de crianças de 0 a 3 anos de idade cuidadas em creches. Rev Bras Crescimento Desenvolv Hum. 2003;13(1):40-52.

26. Chevrie-Muller C, Waltier L, Arabia J, Arabia C, Dellatolas G. Screening by teachers for language and behavior difficulties in 2059 children aged 3.5 years. Rev Epidemiol Sante Publique. 2005:53(6):64557.

27. Hoff E, Tian C. Socioeconomic status and cultural influences on language. J Commun Disord. 2005;38(4):271-8.

28. Souza EM, Grundy E. Promoção da saúde, epidemiologia social e capital social: inter-relações e perspectivas para a saúde pública. Cad Saúde Pública. 2004;20(5):1354-60. 